\title{
A Bayesian Approach to Direction Finding with Parametric Array Uncertainty
}

\author{
MATS VIBERG \\ Dept. of Applied Electronics \\ Chalmers University of Technology \\ $S-41296$ Gothenburg, Sweden
}

\begin{abstract}
With few exceptions, high-resolution source localization algorithms require an exact characterization of the array, including knowledge of the sensor positions, sensor gain/phase response, mutual coupling, and receiver equipment effects. In practice, all such information is inevitably subject to errors. Recently, several different methods have been proposed for alleviating the inherent sensitivity of parametric methods to such modeling errors. The technique proposed herein is related to the class of so-called auto-calibration procedures, but it is assumed that certain prior knowledge of the array response errors is available. The optimal maximum a posteriori (MAP) estimator for the problem at hand is formulated, and a more computationally attractive largesample approximation is derived. In addition, the performance advantage of the algorithm is illustrated by an example involving a linear array mounted on a flexible structure.
\end{abstract}

\section{Introduction}

Model-based methods for array signal processing are based on the assumption that an exact characterization of the array response is available. In practice, of course, such an assumption is quite unreasonable. In fact, the effects of modeling errors typically have at least as great an influence on the total estimation error as do finite sample effects. While most estimation algorithms have been developed with only finite sample effects in mind, alternative techniques that only take model errors into account have been proposed in $[1,2]$. An optimal approach to the problem must account for both sources of errors simultaneously. One idea would be to estimate the unknown parameters of the array response simultaneously with the signal parameters. Such techniques are referred to as auto-calibration methods, and have been proposed in, e.g., $[3,4,5]$. An alternative is to view the modeling errors as random with known second-order statistics. Optimally weighted subspace fitting methods based on this model are derived in [6].

\footnotetext{
${ }^{1}$ This research was supported in part by the Advanced Research Projects Agency of the Department of Defense, monitored by the Air Force Office of Scientific Research under Grant No. F49620-91-C-0096 by the SDIO/IST Pro F Army Research Office under Grant DAAH04-93-G-0029, and by the National Science Foundation under grant MIP-9110112.
}

\author{
A. Lee SWIndlehuRST \\ Dept. of Elec. 8 Comp. Engineering \\ Brigham Young University \\ Provo, UT 84602
}

One drawback associated with auto-calibration techniques is that in many situations, the array response and signal location parameters are not independently identifiable. For instance, it is not possible to estimate both sensor phase characteristics and signal bearing angles. On the other hand, the techniques pursued in $[1,2,6]$ can only give optimal performance for special error models. Herein, we consider a combination of the two approaches. That is, the array perturbation parameters are assumed randorn with known $a$ priori distribution, but they will be estimated in a Bayesian framework along with the signal parameters. The presented results extend the work of $[7,5,8]$. A different approximate maximum a posteriori (MAP) estimator is derived herein using the results of [9], and its asymptotic equivalence with the exact MAP estimator is stated. The performance is illustrated by means of computer simulations of an array with uncertain sensor positions. Additional details (including proofs of statements) can be found in [10].

\section{Problem Formulation}

Consider an array of $m$ sensors, having arbitrary positions and characteristics. Impinging on the array are the waveforms of $d$ narrowband point sources, where $d<m$. The vector of sensor outputs is denoted $\mathbf{x}(t)$ and is modeled by the following familiar equation:

$$
\begin{aligned}
\mathbf{x}(t) & =\left[a\left(\theta_{1}, \rho\right)|\cdots| a\left(\theta_{d}, \boldsymbol{\rho}\right)\right]\left[\begin{array}{c}
s_{1}(t) \\
\vdots \\
s_{d}(t)
\end{array}\right]+\mathbf{n}(t) \\
& =\mathbf{A}(\boldsymbol{\theta}, \boldsymbol{\rho}) \mathbf{s}(t)+\mathbf{n}(t) .
\end{aligned}
$$

The columns of the $m \times d$ matrix $A$ are the so-called $a r$ ray propagation vectors, denoted $a\left(\theta_{i}, \boldsymbol{\rho}\right), i=1, \ldots, d$. These vectors describe the array response to a unit waveform with signal parameter(s) $\theta_{i}$. The above model also allows for unknown "perturbation parameters", collected in the real $n$-vector $\rho=\left[\rho_{1}, \ldots, \rho_{n}\right]^{T}$. This includes structured parameters, such as sensor gain, phase, position, and/or mutual coupling, as well as unstructured (non-physical) parameters. The nominal array response is assumed to be unambiguous; i.e., 
the matrix $\left[a\left(\theta_{1}, \rho_{0}\right), \ldots, a\left(\theta_{m}, \rho_{0}\right)\right]$ has full rank for any collection of distinct parameters $\theta_{1}, \cdots, \theta_{m}$.

Though not necessary, it is assumed in our discussion that $\theta_{i}$ is a real scalar, referred to as the $i^{t h}$ direction-of-arrival (DOA). The components of the $d$ vector $\theta$ are the DOAs of the model, whereas the vector $\theta_{0}$ represents their true values. It is furthermore assumed that the array response parameters in $\rho$ represent small deviations from their known nominal values, collected in the vector $\rho_{0}$. The a priori covariance matrix of the perturbation, denoted by $\mathbf{\Upsilon}$, is also assumed known. The complex $d$-vector $\mathbf{s}(t)$ is composed of the emitter waveforms received at time $t$, and the $m$-vector $\mathbf{n}(t)$ accounts for additive measurement noise.

The signals, noise, and perturbation parameters will all be assumed to be Gaussian random variables when deriving the exact MAP estimator. The covariance of the (zero-mean) signals is denoted by $\mathbf{P}$, and is assumed to be full-rank. The noise is further assumed to be zero-mean, stationary, uncorrelated with the signals, and spatially and temporally white:

$$
\mathrm{E}\left[\mathbf{n}(t) \mathbf{n}^{*}(s)\right]=\sigma^{2} \mathrm{I} \delta_{t, s}, \quad \mathrm{E}\left[\mathbf{n}(t) \mathbf{n}^{T}(s)\right]=0,
$$

where $\delta_{t, s}$ is the Kronecker delta. Given measurements $\mathbf{x}(t)$ for $t=1,2, \ldots, N$, the problem of interest herein is to estimate $\theta$ and to assess the quality of the estimate. The number of signals, $d$, is assumed known.

We are primarily interested in the first-order effects of modeling errors and noise. A mathematically convenient assumption for performing such an analysis is that $\mathbf{\Upsilon}=\overline{\mathbf{Y}} / N$, where $N \gg 1$ and $\overline{\mathbf{Y}}=O(1)$. In the next section, the exact MAP estimator is presented along with a less computationally demanding approximation. The proposed approximate technique is a subspace-based method, in that it relies heavily on the properties of the eigendecomposition of the array covariance. Under the above assumptions, the "covariance matrix" of the array output takes the form

$$
\begin{aligned}
\mathbf{R} & =\lim _{N \rightarrow \infty} \frac{1}{N} \sum_{t=1}^{N} E\left[\mathbf{x}(t) \mathbf{x}^{*}(t)\right] \\
& =\mathbf{A}\left(\boldsymbol{\theta}_{0}, \boldsymbol{\rho}_{0}\right) \mathbf{P} \mathbf{A}^{*}\left(\boldsymbol{\theta}_{0}, \boldsymbol{\rho}_{0}\right)+\sigma^{2} \mathbf{I} .
\end{aligned}
$$

Since the matrix $\mathbf{A P A}{ }^{*}$ has rank $d$ by assumption, $\sigma^{2}$ is an eigenvalue of $\mathbf{R}$ with multiplicity $m-d$, and the corresponding eigenvectors are all orthogonal to $\mathbf{A}$. The eigendecomposition of $\mathbf{R}$ thus takes the form

$$
\mathbf{R}=\sum_{i=1}^{m} \lambda_{i} \mathbf{e}_{i} \mathbf{e}_{i}^{*}=\mathbf{E}_{s} \boldsymbol{\Lambda}_{s} \mathbf{E}_{s}^{*}+\sigma^{2} \mathbf{E}_{n} \mathbf{E}_{n}^{*}
$$

where $\boldsymbol{\Lambda}_{s}$ is a diagonal matrix containing the $d$ largest eigenvalues, and the columns of the $m \times d$ matrix $\mathbf{E}_{s}$ are the corresponding unit-norm eigenvectors. Similarly, the columns of $\mathrm{E}_{n}$ are the $m-d$ eigenvectors corresponding to $\sigma^{2}$. Consistent estimates of the above quantities may be obtained by performing an eigendecomposition of the sample covariance matrix.

\section{Robust MAP Estimation}

It will be assumed that the a priori distribution of $\rho$ is Gaussian with known mean $\rho_{0}$ and covariance matrix $\boldsymbol{\Upsilon}$. The signal parameters, $\boldsymbol{\theta}$, the emitter covariance, $\mathbf{P}$, and the noise variance, $\sigma^{2}$, are all regarded as unknown deterministic parameters. Following [8], the joint MAP estimates of $\boldsymbol{\theta}, \mathbf{P}, \sigma^{2}$ and $\rho$ are then obtained as minimizing arguments of

$$
\begin{aligned}
V_{M A P}\left(\boldsymbol{\theta}, \boldsymbol{\rho}, \mathbf{P}, \sigma^{2}\right)= & V_{M L}\left(\boldsymbol{\theta}, \boldsymbol{\rho}, \mathbf{P}, \sigma^{2}\right) \\
& +\frac{1}{2}\left(\boldsymbol{\rho}-\boldsymbol{\rho}_{0}\right)^{T} \mathbf{\Upsilon}^{-1}\left(\boldsymbol{\rho}-\boldsymbol{\rho}_{0}\right) .
\end{aligned}
$$

Here, $V_{M L}\left(\boldsymbol{\theta}, \boldsymbol{\rho}, \mathbf{P}, \sigma^{2}\right)$ is the negative log-likelihood function. The ML criterion function is known to be separable in $\mathbf{P}$ and $\sigma^{2}$, and may be written in its concentrated form

$$
V_{M L}(\boldsymbol{\theta}, \boldsymbol{\rho})=N \log \left|\mathbf{A} \hat{\mathbf{P}} \mathbf{A}^{*}+\dot{\sigma}^{2} \mathbf{I}\right|+\text { const }
$$

after substituting appropriate $\mathrm{ML}$ estimates for $\mathbf{P}$ and $\sigma^{2}[11]$. Clearly, $V_{M A P}\left(\boldsymbol{\theta}, \boldsymbol{\rho}, \mathbf{P}, \sigma^{2}\right)$ is also separable in $\mathbf{P}$ and $\sigma^{2}$, and ignoring constant terms the concentrated MAP criterion function is

$V_{M A P}(\boldsymbol{\theta}, \boldsymbol{\rho})=V_{M L}(\boldsymbol{\theta}, \boldsymbol{\rho})+\frac{1}{2}\left(\boldsymbol{\rho}-\boldsymbol{\rho}_{0}\right)^{T} \mathbf{Y}^{-1}\left(\boldsymbol{\rho}-\boldsymbol{\rho}_{0}\right)$.

This can be interpreted as a regularized ML criterion That is, the effect of the prior distribution is to force $\hat{\rho}_{M A P}$ to be close to the nominal value, $\rho_{0}$. If the perturbation parameters are identifiable, this effect is diminished as the number of snapshots, $N$, increases. In such cases, the MAP estimate has the same asymptotic properties as the ML estimate (i.e., the pure autocalibration technique). However, in many applications of interest, $\rho$ cannot be consistently estimated along with the signal parameters. In these situations the prior distribution has a crucial influence on the asymptotic properties of the estimates of both $\theta$ and $\rho$.

\subsection{The MAP-NSF Method}

It has been assumed that the signal covariance matrix has full rank; i.e., the signals are non-coherent. Then it is known [11] that in the absence of model errors, the ML criterion is asymptotically equivalent to the following noise subspace fitting (NSF) criterion

$$
V_{N S F}=N \operatorname{Tr}\left\{\mathbf{A}^{*} \hat{\mathbf{E}}_{n} \hat{\mathbf{E}}_{n}^{*} \mathbf{A} \hat{\mathbf{U}}\right\},
$$

where $\hat{\mathbf{U}}$ denotes a consistent estimate of the matrix

$$
\mathbf{U}=\sigma^{-2} \mathbf{A}^{\dagger} \mathbf{E}_{s} \tilde{\mathbf{\Lambda}}^{2} \mathbf{\Lambda}_{s}^{-1} \mathbf{E}_{s}^{*} \mathbf{A}^{\dagger *}=\sigma^{-2} \mathbf{P} \mathbf{A}^{*} \mathbf{R}^{-1} \mathbf{A} \mathbf{P}
$$


and $\tilde{\boldsymbol{\Lambda}}=\boldsymbol{\Lambda}_{s}-\sigma^{2} \mathbf{I}$. By standard first order arguments, this implies that the MAP estimate is asymptotically (for large $N$ ) equivalent to the minimizing arguments of the following criterion function

$$
V_{N S F}(\theta, \rho)+\frac{1}{2}\left(\rho-\rho_{0}\right)^{T} \mathbf{\Upsilon}^{-1}\left(\rho-\rho_{0}\right)
$$

This criterion depends on its parameters in a simpler way than the exact MAP criterion (8). However, it still requires a non-linear minimization over both $\theta$ and $\rho$.

Using standard formulas for the vec $(\cdot)$ operator (vectorization of a matrix by stacking its columns) and the Kronecker product $\otimes$, the NSF criterion can be rewritten using

$$
\begin{aligned}
\operatorname{Tr}\left\{\mathbf{A}^{*} \hat{\mathbf{E}}_{n} \hat{\mathbf{E}}_{n}^{*} \mathbf{A} \hat{\mathbf{U}}\right\} & =\operatorname{vec}\left\{\left(\mathbf{A}^{*} \hat{\mathbf{E}}_{n} \hat{\mathbf{E}}_{n}^{*}\right)^{\mathrm{T}}\right\}^{\mathbf{T}} \operatorname{vec}\{\mathbf{A} \hat{\mathbf{U}}\} \\
& =\mathbf{a}^{*} \hat{\mathbf{M}} \mathbf{a},
\end{aligned}
$$

where $\mathbf{a}=\operatorname{vec}(\mathbf{A})$ and $\hat{\mathbf{M}}=\hat{\mathbf{U}}^{T} \otimes\left(\hat{\mathbf{E}}_{n} \hat{\mathbf{E}}_{n}^{*}\right)$. Next, the vectorized steering matrix is approximated locally around $\rho_{0}$ as

$$
\mathbf{a}=\mathbf{a}(\boldsymbol{\theta}, \boldsymbol{\rho}) \approx \mathbf{a}_{0}+\mathbf{D} \boldsymbol{\rho} \tilde{\boldsymbol{\rho}},
$$

where $\mathbf{a}_{0}=\mathbf{a}\left(\theta, \rho_{0}\right), \tilde{\boldsymbol{\rho}}=\boldsymbol{\rho}-\boldsymbol{\rho}_{0}$, and

$$
\mathbf{D}_{\rho}=\left.\frac{d \mathbf{a}(\theta, \rho)}{d \rho}\right|_{\theta, \rho_{0}} .
$$

Note that, when evaluated at $\rho_{0}$, the derivative of a with respect to $\theta$ or $\rho$ is identical to that of $a_{0}+D_{\rho} \tilde{\rho}$. It follows that the minimizing arguments of (12) are asymptotically identical to the estimates obtained by minimizing the following approximate MAP-NSF criterion with respect to $\theta$ and $\rho$ :

$$
\left(\mathbf{a}_{0}+\mathbf{D}_{\boldsymbol{\rho}} \tilde{\boldsymbol{\rho}}\right)^{*} \hat{\mathbf{M}}\left(\mathbf{a}_{0}+\mathbf{D}_{\boldsymbol{\rho}} \tilde{\boldsymbol{\rho}}\right)+\frac{1}{2} \tilde{\boldsymbol{\rho}}^{T} \overline{\mathbf{\Upsilon}}^{-1} \tilde{\boldsymbol{\rho}},
$$

where we have normalized by $N$.

Since the criterion function in (15) is quadratic in $\tilde{\rho}$, we easily obtain the minimum with respect to $\tilde{\rho}$ (for fixed $\theta$ ) as

$$
\hat{\boldsymbol{\rho}}_{M A P-N S F}=\rho_{0}-\boldsymbol{\Gamma}^{-1} \mathbf{f},
$$

where

$$
\begin{aligned}
\mathbf{\Gamma} & =\operatorname{Re}\left\{\mathbf{D}_{\boldsymbol{\rho}}^{*} \hat{\mathbf{M}} \mathbf{D}_{\boldsymbol{\rho}}+\frac{1}{2} \overline{\mathbf{Y}}^{-1}\right\} \\
\mathbf{f} & =\operatorname{Re}\left\{\mathbf{D}_{\boldsymbol{\rho}}^{*} \hat{\mathbf{M}} \mathbf{a}_{0}\right\}
\end{aligned}
$$

Substituting (16) into (15) leads to the following separated criterion function

$$
\mathbf{a}_{0}^{*} \hat{\mathbf{M}} \mathbf{a}_{0}-\mathbf{f}^{T} \boldsymbol{\Gamma}^{-1} \mathbf{f} .
$$

Note that $\boldsymbol{\Gamma}$ and $\mathbf{f}$ depend on $\boldsymbol{\theta}$ through $\mathbf{D} \boldsymbol{\rho}$, and in principle $\mathbf{M}$ also depends on $\boldsymbol{\theta}$ through $\mathbf{U}$. However, it will be assumed that a consistent estimate of $\theta$ is available to form the estimates $\hat{\mathbf{M}}, \hat{\mathbf{f}}$ and $\hat{\boldsymbol{\Gamma}}$. We thus finally arrive at the proposed approximate MAP-NSF estimate, defined by

$$
\hat{\boldsymbol{\theta}}_{M A P-N S F}=\arg \min _{\boldsymbol{\theta}}\left\{\mathbf{a}_{0}^{*} \hat{\mathbf{M}} \mathbf{a}_{0}-\hat{\mathbf{f}}^{T} \hat{\mathbf{\Gamma}}^{-1} \hat{\mathbf{f}}\right\} .
$$

As shown in [10], the approximations made in $\hat{\boldsymbol{\Gamma}}, \hat{\mathbf{f}}$ and $\hat{\mathbf{M}}$ do not change the asymptotic properties of the final estimate, i.e., $\hat{\boldsymbol{\theta}}_{M A P-N S F}$ is asymptotically identical to the exact MAP estimator.

\section{A Simulation Example}

As an application of the general perturbation model considered herein, we study a model suitable for arrays mounted on a flexible structure. The array is assumed to be planar, although an extension to the threedimensional case is straightforward. It is assumed that the distance between the sensors is known and fixed. For simplicity, we approximate the flexible structure using a piecewise linear model, as illustrated in Figure 1 . This approximation is reasonable for small perturbations, which is the case of interest herein. The nominal

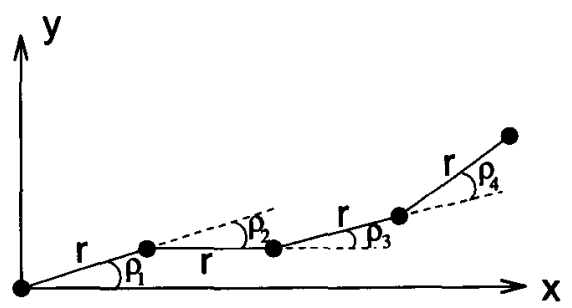

Figure 1: An array mounted on a flexible structure

array is assumed to be a uniform linear array (ULA) of $m=10$ sensors, oriented along the $x$-axis of a coordinate system with its origin at the first sensor. The interelement spacing ( $r$ in the figure) is fixed at a half wavelength. In many cases of practical interest, there may be physical constraints that relate the incremental angles, thus leading to a perturbation model with fewer parameters (for example a polynomial model). However, here we assume that $\rho_{i}, i=1, \ldots, m-1$, are independent zero-mean Gaussian random variables with variance $\nu^{2}$. This is a reasonable model, e.g., for a towed array.

The perturbed array receives the waveforms of two uncorrelated signal sources located at $\theta_{1}=85^{\circ}$ and $\theta_{2}=90^{\circ}$, where $\theta$ is measured counter-clockwise rel- 
ative to the $x$-axis. 'The MAP-NSF method estimates the DOAs and simultaneously calibrates the $\rho$ parameters as described in Section 3.1. For comparison, we also include the WSF [12] estimates using the nominal ULA parametrization. The signal-to-noise ratio (SNR) is fixed at $10 \mathrm{~dB}$ for both signals. In the first experiment, the perturbation variance $\nu^{2}$ is varied, whereas the number of snapshots in each batch is fixed at $N=1000$. Figure 2 displays the theoretical and empirical RMS errors of the estimate of $\theta_{1} \mathrm{vs}$ $\nu$ (both axes in degrees). The theoretical results are obtained using results presented in [10]. The empirical results are calculated from 512 independent MonteCarlo runs. As seen from the figure, the theoretical

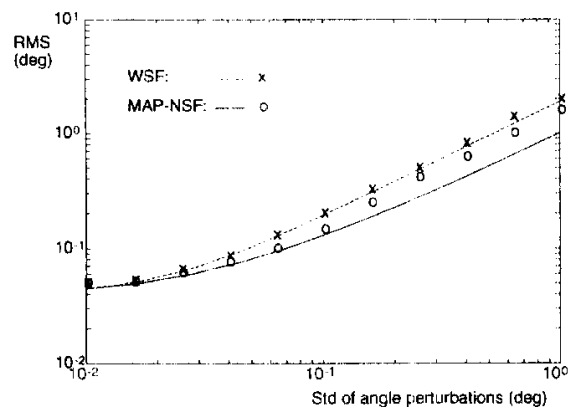

Figure 2: Theoretical and empirical RMS crror of the DOA es timates versus the standard deviation of the incremental sensar angle perturbations.

RMS error of the MAP-NSF estimate (i.e., the (RRB) is slightly but clearly smaller than that of the WSF estimate, the difference being more pronounced for large $v$. However, the CRB appears to be difficult to reach in practice. The empirical results agree well with the theory only for $\nu \leq 0.1^{\circ}$. For smaller values of $\nu$, the performance difference between WSF and MAP-NSF hecomes insignificant.

As suggested by the analysis in [10], the CRH wil be reached and MAP-NSF will outperform WSF when $t^{2}$ is of order $O(1 / N)$. Thus, in the next experiment the number of snapshots is varied from $N=100$ to $N=10000$, whereas $\nu^{2}$ is simultaneosly decreased is $\nu^{2}(N)=c / N$, where $c$ is determined such that $v(1000)=0.1^{\circ}$. Figure 3 shows the theoretical and empirical RMS errors for $\hat{\theta}_{1}$ versus the number of snapshots. In this case, the empirical RMS errors of the MAP-NSF estimates approaches the CRB for $V>1000$ and $\nu<0.1^{\circ}$, and the R.MS error of the WSF estimates is about $50 \%(3.5 \mathrm{~d} \mathrm{~B})$ higher.

\section{References}

[1] A. Swindlehurst and T. Kailath, "A Performance Analysis

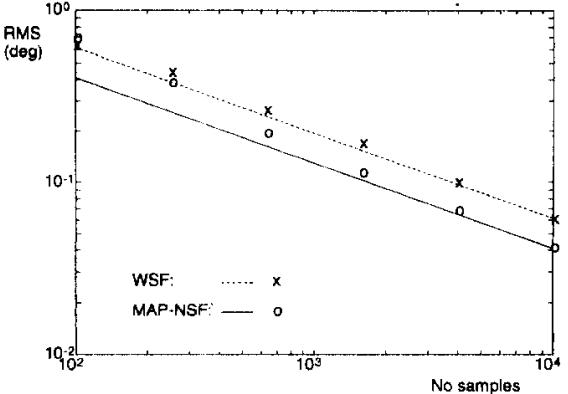

figure 3: Theoretical and empirical RMS error of the DOA estimates versus the number of snapshots.

of Subspace-Based Methods in the Presence of Model Errors - Part I: The MUSIC Algorithm", IEEE Trans. SP, SP40(7):1758-1774, July 1992 .

[2] A. Swindlehurst and T. Kailath, "A Performance Analysis of Subspace-Based Methods in the Presence of Model Errors - Part II: Multidimensional Algorithms", IEEE Trans. on SP, SP-41(9):1277-1308, Sept. 1993.

[3] A. Paulraj and T, Kailath, "Direction-of-Arrival Estimation by Eigenstructure Methods with Unknown Sensor Gain and Phase", In Proc. IEEE ICASSP, pages 17.7.1-17.7.4, Tampa, Fla., March 1985.

[4] B. Friedlander and A. J. Weiss, "Eigenstructure Methods for Direction Finding with Sensor Gain and Phase Uncertainties", In Proc. ICASSP, pages 2681-2684, 1988.

[5] A. J. Weiss and B. Friedlander, "Array Shape Calibration Using Sources in Unknown Locations - A Maximum Likelihood Approach", IEEE Trans. on ASSP, 37(12):19581966, Dec. 1989.

[6] M. Viberg and A. Swindlehurst, "Analysis of the Combined Effects of Finite Samples and Model Errors on Array Processing Performance", In Proc. ICASSP, Minueapolis, MN, April 1993.

[7] Y. Rockah and P. M. Schultheiss, Array shape calibration using sources in unknown locations - Part TI: Near-field sources and estimator implementation, IEEE Trans. on ASSP, 35:724-735, June 1987.

[8] B. Wahlberg, B. Ottersten, and M. Viberg, "Riobust Signal Parameter Estimation in the Presence of Array Perturbations", In Proc. ICASSP. Toronto, Canada, May 1991.

[9] P. Stoica and K. Sharman, "Maximum Likelihood Methods for Direction-of-Arrival Estimation", IEEE Trans. ASSP, ASSP-38:1132-1143, July 1990 .

[10] M. Viberg and A. Swindlehurst, "A Bayesian Approach to Auto-Calibration for Parametric Array Signal Processing", IEEE Trans. on SP, 1994, (to appear).

[11] B. Ottersten, M. Viberg, P. Stoica, and A. Nehorai, Exact and large sample ML techniques for parameter estimation and detection in array processing. In Haykin, Litva, and Shepherd, editors, Radar Array Processing, pages 99-151. Springer-Verlag, Berlin, 1993.

[12] M. Viberg and B. Ottersten, "Sensor Array Processing Based on Subspace Fitting", IEEE Trans. SP, SP39(5): $1110-1121$. May 1991 . 